\title{
DOCENCIA VIRTUAL EN UNIVERSIDADES PRESENCIALES: EXPERIENCIA EN LA UNIVERSIDAD DE CASTILLA-LA MANCHA
}

\author{
(VIRTUAL EDUCATION IN TRADITIONAL UNIVERSITIES: EXPERIENCE IN THE UNIVERSITY OF \\ CASTILE-LA MANCHA)
}

\author{
Juan Antonio Mondéjar \\ José Mondéjar Jiménez \\ Manuel Vargas Vargas \\ Universidad de Castilla-La Mancha (España)
}

\section{RESUMEN}

La docencia virtual se ha convertido en una herramienta habitual en nuestras universidades presenciales. Las nuevas metodologías de aprendizaje para los estudiantes, junto a nuevas técnicas pedagógicas al amparo del nuevo Espacio Europeo de Educación Superior, han posibilitado que tanto la red Internet como las nuevas tecnologías de la información y la comunicación (NTIC) se conviertan en nuevas opciones de enseñanza que combinan diferentes recursos pedagógicos, haciendo compatible la presencialidad y la no presencialidad. Estos nuevos sistemas de enseñanza son capaces de controlar los tiempos de aprendizaje, así como la cantidad de trabajo desarrollado por el alumno, lo que aporta una mayor información a los procesos de enseñanza-aprendizaje.

Nos encontramos ante una verdadera transformación de la docencia superior en las universidades presenciales: la buena integración de las NTIC en la actividad docente abre nuevas posibilidades para los contenidos que forman parte de la actividad formativa, sin perder de vista las nuevas capacidades que se ofrecen en este ámbito a los propios estudiantes.

El presente trabajo analiza la experiencia empírica desarrollada por los autores en la Universidad de Castilla-La Mancha, desde la plataforma "Red-C@mpus" hacia el entorno virtual de aprendizaje denominado "Campus Virtual". En ambos casos, tanto estudiantes como profesores interactúan de forma asincrónica en un entorno que tiene como finalidad facilitar el proceso de enseñanza-aprendizaje que posibilite culminar los propósitos propuestos.

Palabras clave: entorno virtual de aprendizaje, Red-C@mpus, campus virtual, e-learning. 


\begin{abstract}
Virtual teaching has become a common tool in our traditional universities. The new learning methodologies for the students, together with new pedagogical techniques in the context of the new European Space for Higher Education, have meant that both the Internet and the new information and communications technologies (ICT) have become options for teaching that combine various pedagogical resources and make traditional classroom-based teaching and the new virtual teaching compatible. These new teaching systems can control both the time students spend learning and the amount of work they do, thereby offering valuable information to the teaching-learning processes.

Higher education in traditional universities is being truly transformed: the successful integration of ICT into the teaching opens up new possibilities for the contents on the syllabus, and offers the students themselves the chance to gain new skills in this area.

The current work analyses the empirical experiment that the authors have carried out in the University of Castile-La Mancha, from the "Red-C@mpus" platform to the virtual learning environment called "Campus Virtual". In both cases, students and lecturers interact asynchronously in an environment that is designed to facilitate the teaching-learning process and ensure that the proposed objectives can be achieved.
\end{abstract}

Key words: virtual environment of learning, Red-C@mpus, virtual campus, e-learning.

Las universidades de toda Europa viven unos momentos de cambio en la actualidad, con motivo de su adecuación al nuevo Espacio Europeo de Educación Superior (EEES). La adaptación de todos los planes de estudio, titulaciones y enseñanzas al nuevo sistema ECTS (European Transfer Credits System) supone un verdadero reto de futuro para toda la institución, especialmente para profesores y estudiantes.

En este sentido, la docencia virtual se ha convertido en una herramienta habitual en nuestras universidades presenciales, sobre la base de las nuevas metodologías de aprendizaje para los estudiantes y las nuevas técnicas pedagógicas que requiere el nuevo EEES.

Las nuevas Tecnologías de la Información y la Comunicación (TIC) juegan un papel esencial en la docencia virtual, por lo que el importante desarrollo que ha seguido el acceso a líneas ADSL de alta velocidad de acceso a Internet, así como la proliferación de conexión en los hogares españoles, han posibilitado el rápido desarrollo de metodologías de enseñanza-aprendizaje basadas exclusivamente en entornos virtuales y sistemas e-learning: por ejemplo, la Universidad Oberta de Cataluña (UOC) desarrolla, desde su fundación en 1996, toda su docencia de títulos 
oficiales universitarios de grado, posgrado y master exclusivamente a través de Internet (www.uoc.edu), considerándose su Campus Virtual como uno de los más avanzados y completos del panorama universitario a nivel internacional. Es la primera universidad europea totalmente virtual.

Al igual que en el ejemplo de la UOC, los nuevos retos de la enseñanza universitaria en España han llevado a prácticamente todas las universidades presenciales a desarrollar plataformas de acceso virtual y de e-learning: por ejemplo, el Grupo 9 de Universidades (G-9) es una asociación sin ánimo de lucro que reúne a las universidades públicas que son únicas en sus respectivas Comunidades Autónomas (Castilla-La Mancha, Extremadura, Oviedo, Cantabria, País Vasco, Pública de Navarra, Zaragoza, La Rioja e Illes Balears) con el fin de potenciar sus actividades académicas.

Una de sus actividades más importantes es el desarrollo de un Campus Virtual Compartido (CVC), proyecto de compartición de asignaturas a través de Internet. Este CVC se ve complementado por el proyecto Profesores Invitados por Red, por el que sus alumnos pueden asistir a través de videoconferencia a una clase impartida por un docente de otra universidad que complete el contenido del profesor oficial de la asignatura. De este modo, cualquier alumno puede acceder, desde un ordenador con conexión a Internet, a una asignatura ofertada en cualquiera de las nueve universidades participantes, con un desarrollo íntegramente a través de la red.

El presente trabajo realiza, en este sentido, una breve introducción sobre las herramientas virtuales utilizadas en la Universidad de Castilla-La Mancha: desde sus inicios a través de la plataforma Red-C@mpus hasta el recientemente instaurado Campus Virtual; a continuación se analiza la aplicación de ambas plataformas en diversas asignaturas y, por último, se destacan las principales conclusiones y líneas de investigación futura derivadas del trabajo.

\section{E-LEARNING EN LA UNIVERSIDAD DE CASTILLA-LA MANCHA}

La Universidad de Castilla-La Mancha (UCLM) es una universidad multicampus que lleva a cabo su actividad docente e investigadora sobre la base de cuatro campus universitarios ubicados en otras tantas capitales de provincia de la comunidad autónoma, a los que se añaden tres centros universitarios en las ciudades más significativas de la región. 
Desdesusinicios,laUniversidaddeCastilla-LaManchahaapostadodecididamente por el uso de las nuevas tecnologías de la información y la comunicación (NTIC) tanto en el espacio de enseñanza a los estudiantes como en las áreas de gestión administrativa y en el proceso de docencia e investigación del profesorado.

Una de las novedades más importantes en este sentido está constituida por la incorporación de las nuevas tecnologías de la información y de la comunicación (TIC) a la enseñanza tradicional, por medio de las cuales debemos ser capaces de reconducir la docencia y adecuarnos a los nuevos escenarios de educación, especialmente motivados por el Espacio Europeo de Educación Superior. En la actualidad, de forma paulatina, se va generalizando la incorporación al ámbito universitario de estas tecnologías.

De estos novedosos sistemas de enseñanza-aprendizaje, el e-learning destaca por ser capaz de integrar el uso de las nuevas tecnologías y elementos didácticos tradicionales para conseguir obtener el mayor partido posible a las nuevas tecnologías. Entre las principales ventajas de este tipo de aprendizaje, tal y como señalan González, López y Chasco (2005), destacan:

- Posibilidad de asistencia a clase de forma remota.

- Posibilita un espacio virtual de reunión para la realización de trabajos.

- Es una fuente de reducción de costes.

- $\quad$ Permite un aprendizaje más dinámico.

En esta misma línea se sitúan las características específicas del e-learning que lo diferencian de otras modalidades de educación a distancia, a saber (Acosta Lugo, 2004):

- Distancia física.

- Trabajo individual o en grupo.

- Horario flexible.

- Llega a un número mayor de destinatarios.

- $\quad$ Ritmo de aprendizaje individualizado.

- Interactivo y multimedia.

- Multiplicidad de expertos.

- Evaluación on-line. 
Este nuevo entorno virtual de aprendizaje responde favorablemente a los factores relevantes para la creación de comunidades de aprendizaje (Valverde, 2002), así, el e-learning es capaz de suprimir el contacto presencial por el contacto virtual, puede estar sujeto a una serie de normas o reglas de funcionamiento fijadas por los administradores del mismo, se puede controlar la cantidad de información suministrada en todo momento y, por último, el tamaño de los grupos - que era una variable prioritaria en la enseñanza presencial- pasa a un segundo plano, y sólo será relevante para el hecho de no poder realizar un seguimiento por parte del profesor de todos sus alumnos.

\section{RED-C@MPUS}

La Universidad de Castilla-La Mancha implantó, a partir del curso académico 2000/2001, la aplicación informática Red-C@mpus junto a otras Universidades de nuestro país (Salamanca, La Rioja, La Laguna, Rey Juan Carlos, Cádiz, entre otras).

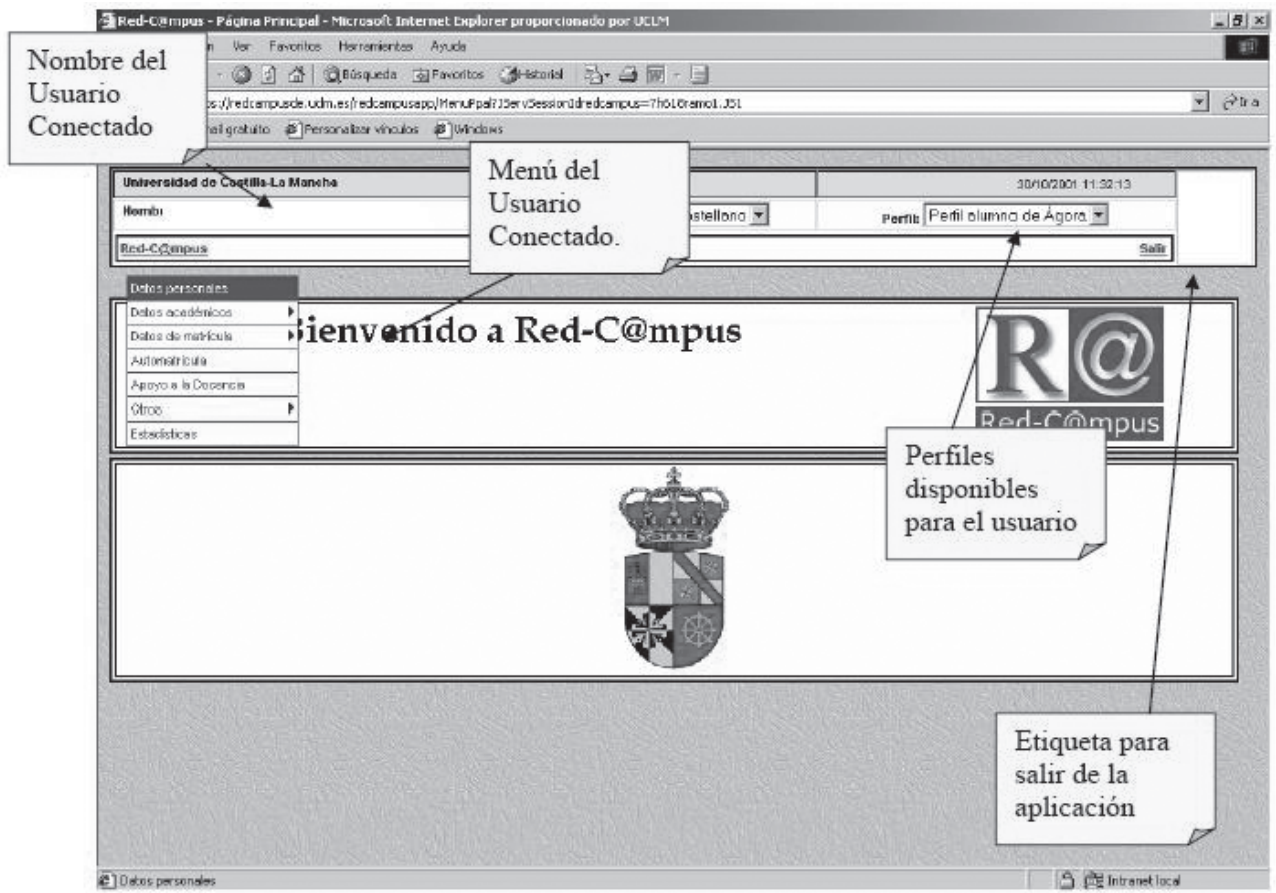

Figura 1.Portada de Red-C@mpus en la UCLM 
El propósito de esta herramienta se centra en el apoyo a la Universidad en todas sus actividades. De esta forma, Red-C@mpus se concibió como un proyecto encaminado a favorecer la comunicación y la interacción no sólo dentro de la propia Universidad, sino también entre ella y la ciudadanía, eliminando las barreras de tiempo y espacio que muchas veces actúan. Se trata de poder acceder a todos aquellos datos propios de la docencia y la gestión universitaria a través de Internet, cajeros automáticos, etc. Nos permite trabajar con todas las aplicaciones existentes de docencia (Mondéjar, Gómez y Lorenzo, 2006).

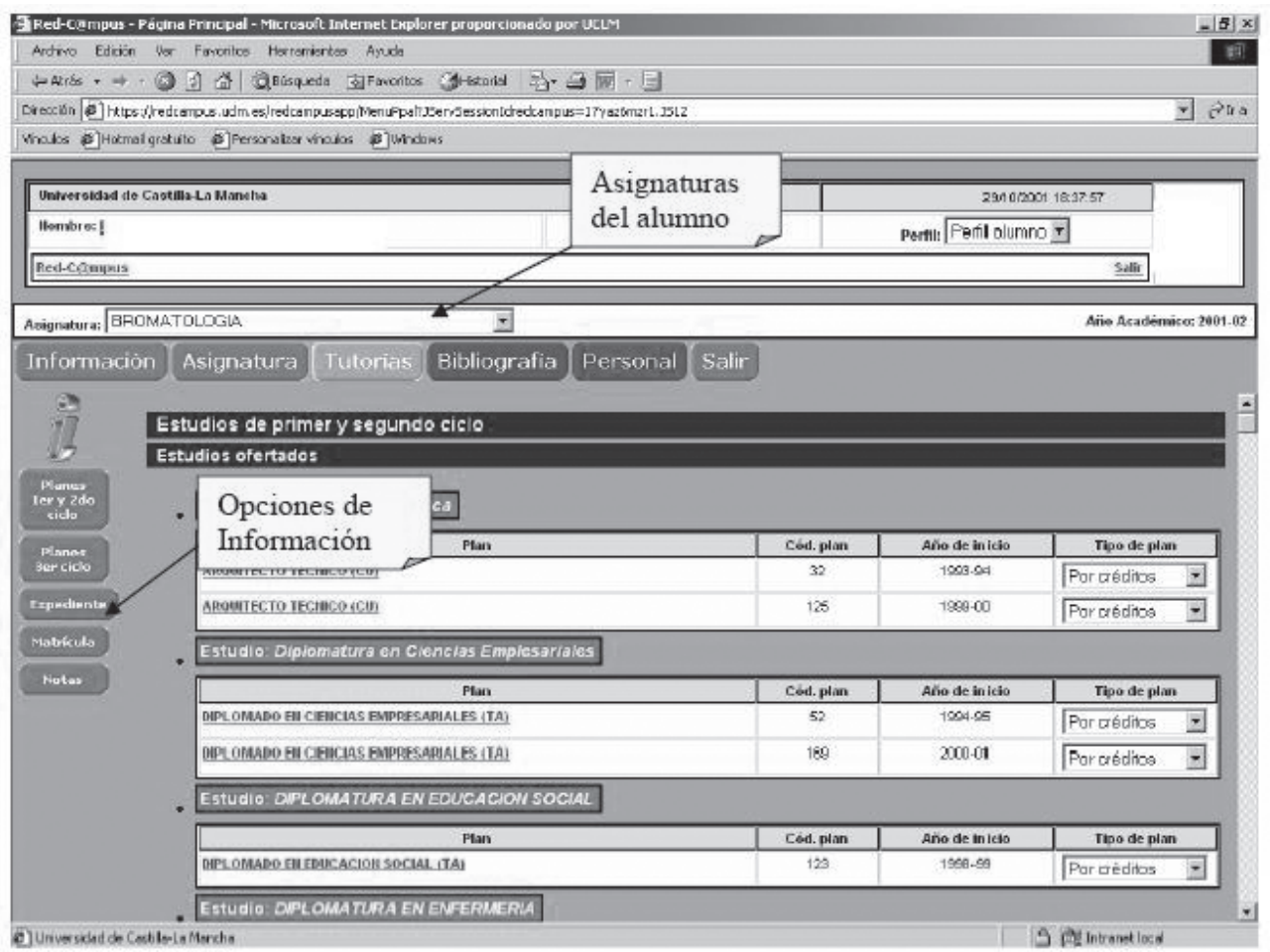

Figura 2. Estructura jerárquica Red-C@mpus

Las posibilidades que ofrece, en este sentido, la plataforma Red-C@ampus son las siguientes:

- Datos personales.

- Expediente.

- Datos matrícula. 
- Calendario académico.

- Configuración del navegador.

- Buzón alumno.

- $\quad$ Solicitud Becas.

Existe un apartado dedicado exclusivamente al e-learning denominado apoyo a la docencia, que consta de los siguientes apartados:

- Información (sobre plan de estudios).

- Asignatura (sobre cada asignatura en particular).

- Tutorías (Tutoría electrónica y preguntas frecuentes).

- Bibliografía (donde se incluyen todos los materiales de la asignatura).

- Personal (para el cambio y gestión de claves).

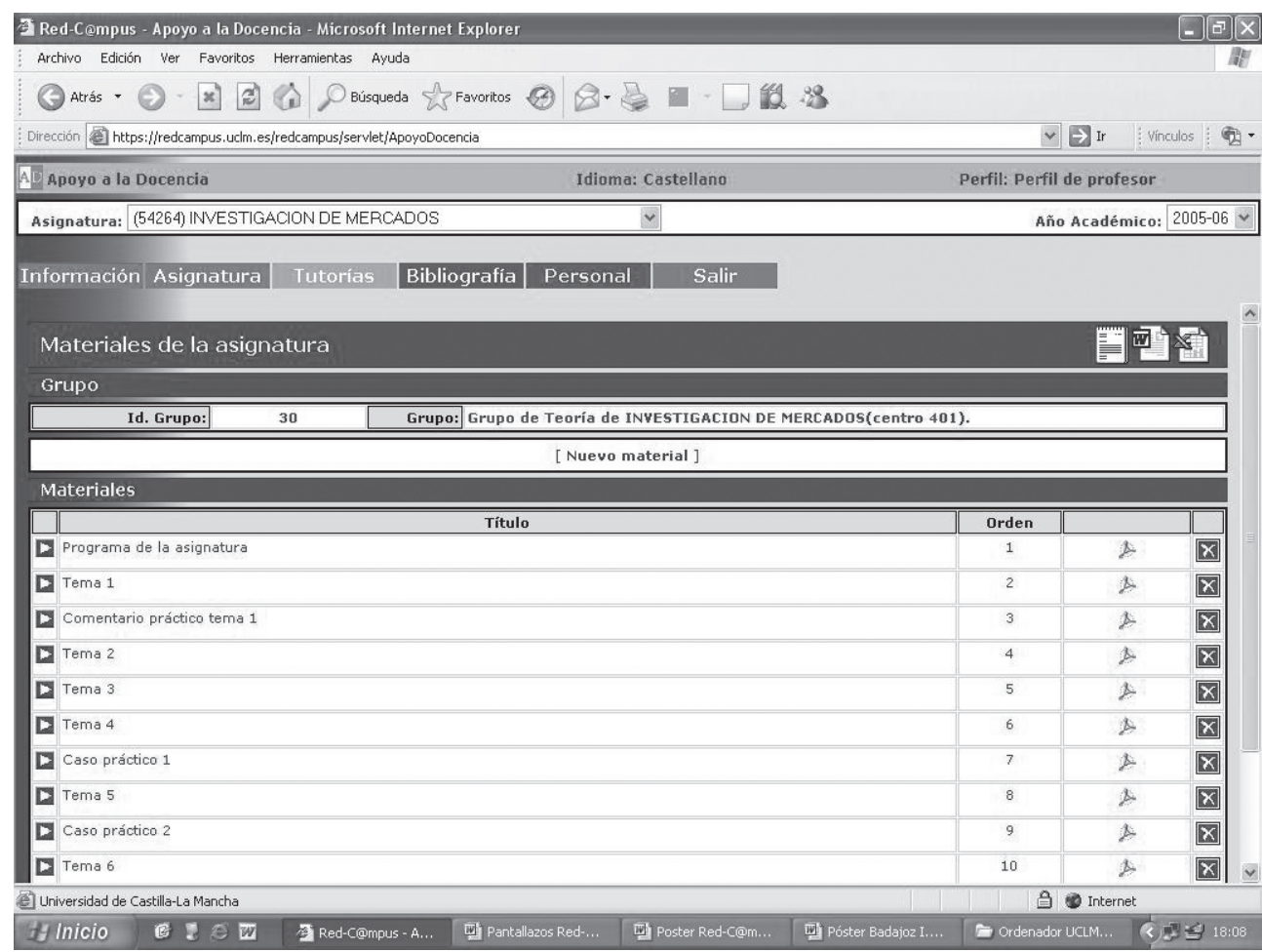

Figuras 3. Ejemplos de materiales asignatura 


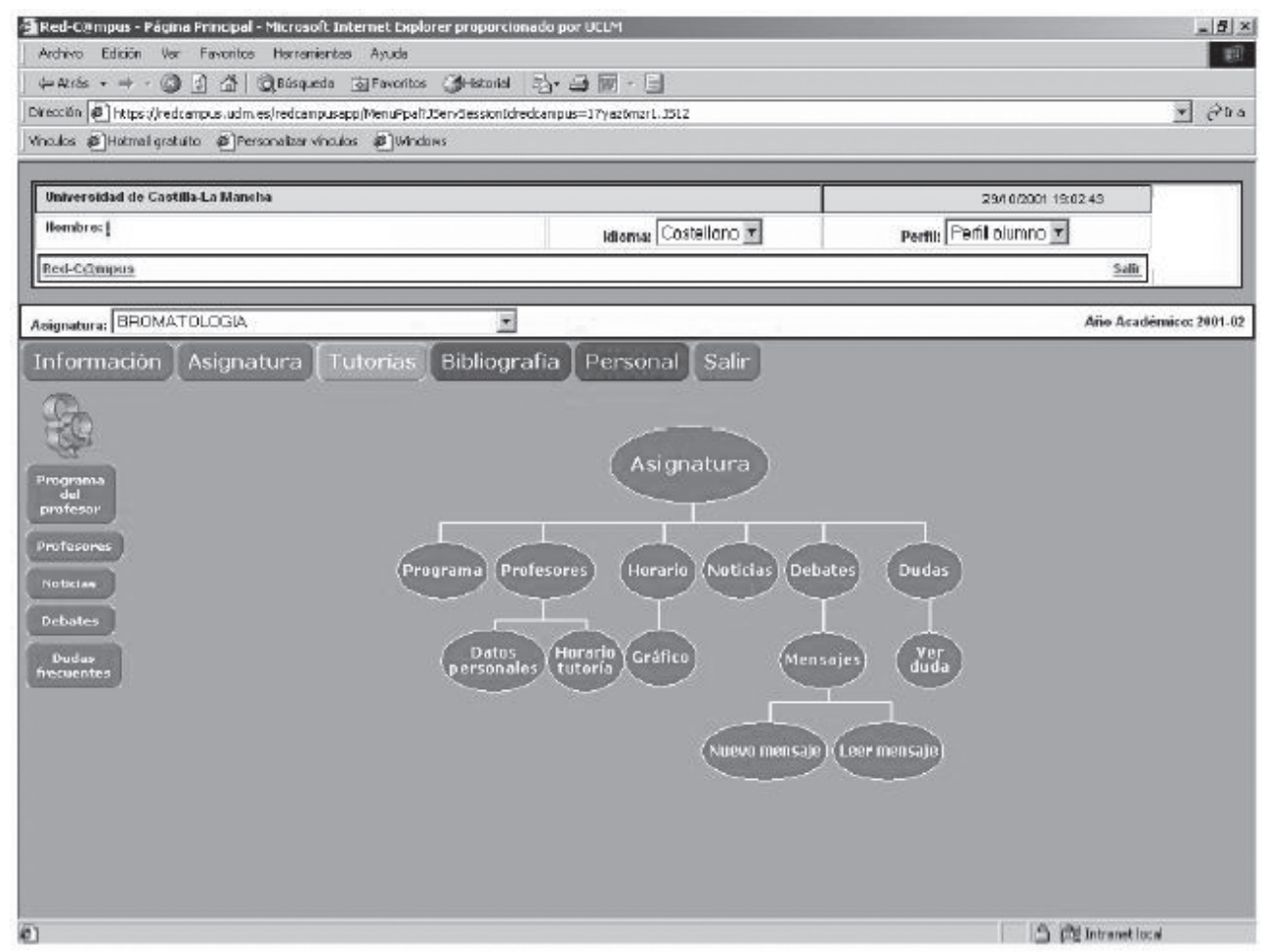

Figuras 4. Ejemplos de materiales asignatura

En definitiva, la iniciativa Red-C@mpus finalizará su aplicación en la Universidad de Castilla-La Mancha en el curso académico 2007/2008, de tal forma que será completamente sustituida por las nuevas herramientas que configuran el Campus Virtual, a las que hacemos referencia en el epígrafe siguiente, y dejará de funcionar tras ocho cursos académicos en los que se ha configurado como una herramienta imprescindible para el correcto desarrollo docente y administrativo, los últimos cuatro simultaneados con el nuevo Campus Virtual.

\section{Campus Virtual}

El Campus Virtual es una herramienta desarrollada por el Grupo 9 de Universidades (G-9), una asociación sin ánimo de lucro ${ }^{1}$ que reúne a las universidades públicas que son únicas en sus respectivas Comunidades Autónomas (Castilla-La Mancha, Extremadura, Oviedo, Cantabria, País Vasco, Pública de Navarra, Zaragoza, La Rioja e Illes Balears) con el fin de potenciar sus actividades académicas. 
En los cursos 2004/2005, 2005/2006 y 2006/2007 se ha utilizado la herramienta Campus Virtual dentro de la Universidad de Castilla-La Mancha de manera voluntaria por parte de los profesores y - sobre todo y más importante- de forma experimental. Así, esta herramienta ha convivido en la Universidad de Castilla-La Mancha con el anterior proyecto virtual denominado Red-C@mpus, ${ }^{2}$ que ha sido la única herramienta institucional utilizada por defecto por profesores y alumnos de la UCLM - como hemos planteado en el epígrafe anterior- aunque está prevista su sustitución completa por Campus Virtual en el próximo curso 2008/2009.

Concretamente, en la Facultad de Ciencias Sociales de Cuenca se inició un Programa de Entrenamiento de Créditos ECTS a partir del curso académico 2004/2005, al que se incorporaron de forma voluntaria la mayor parte de las asignaturas optativas. A raíz de esta experiencia, en el siguiente curso académico 2005/2006, se iniciaron tres Proyectos de Innovación Docente para cada una de las titulaciones que integran la Facultad -Administración y Dirección de Empresas, Derecho y Relaciones Laborales-, financiados por el Vicerrectorado de Convergencia Europea y Ordenación Académica de la Universidad. En los tres casos, todas las asignaturas de primer curso de las tres titulaciones se incorporaron a esta experiencia, considerando las nuevas técnicas pedagógicas al amparo del Espacio Europeo de Educación Superior.

La iniciativa ha tenido su continuidad en el curso 2006/2007, con la incorporación de las asignaturas de segundo curso. Para el nuevo curso 2007/2008 está prevista la ampliación de los Proyectos de Innovación Docente para las asignaturas de tercer curso, completando de este modo el primer ciclo de las licenciaturas en Administración y Dirección de Empresas y Derecho y toda la diplomatura en Relaciones Laborales. De manera voluntaria para este curso se ha posibilitado la incorporación del resto de asignaturas de segundo ciclo, así como las materias optativas que todavía no formaban parte de los proyectos. 


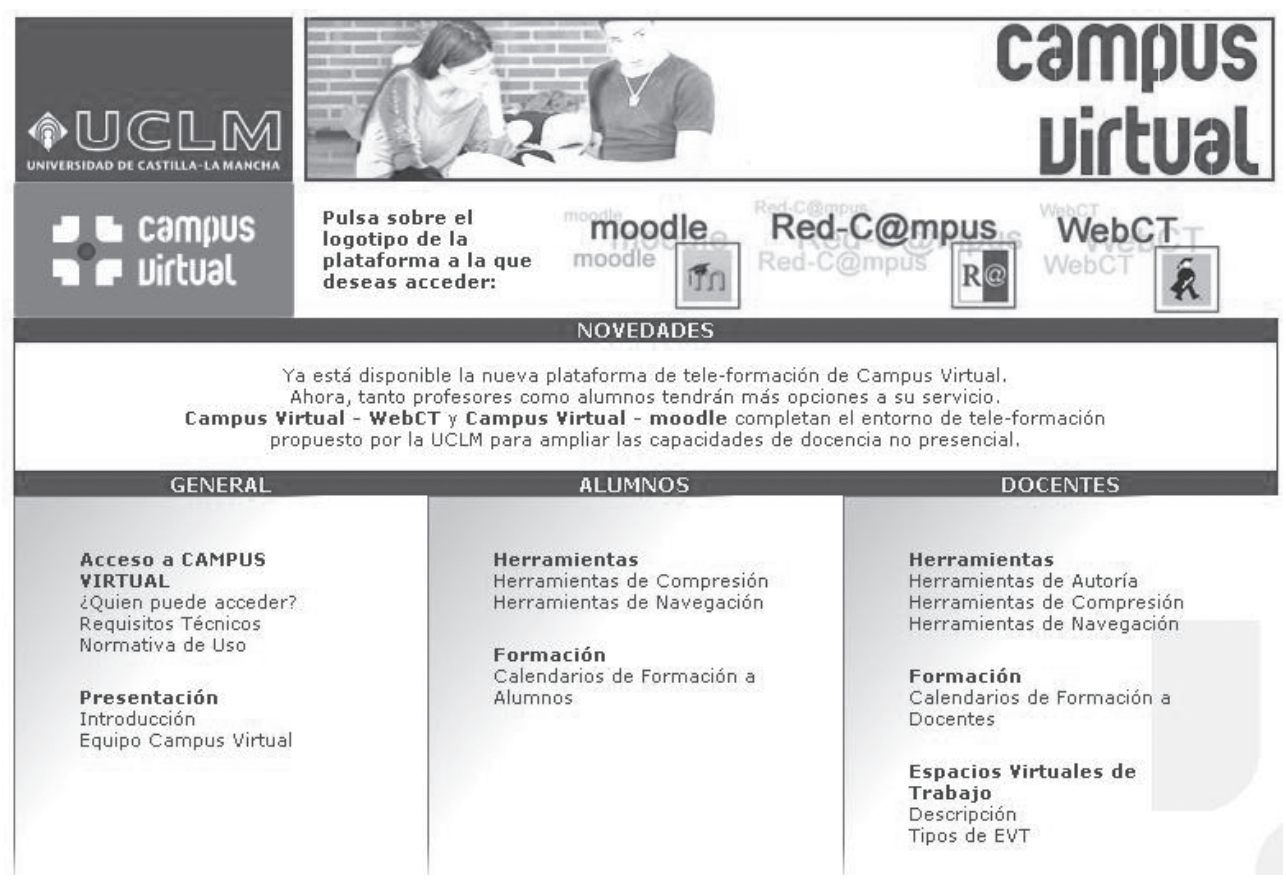

Figura 5. Portada de Campus Virtual en la UCLM ${ }^{3}$

En la página de entrada a Campus Virtual (figura 5), podemos elegir entre el sistema de acceso al mismo, ya que actualmente la UCLM ofrece tres posibilidades: el acceso al anterior sistema Red-C@mpus, así como a los nuevos WebCTy Moodle.

Además, la herramienta posibilita encontrar la siguiente información, dividida en tres apartados:

- General: quién puede acceder, requisitos técnicos, normativa de uso y presentación y equipo de Campus Virtual.

- Alumnos: herramientas de compresión, herramientas de navegación y calendario de formación a alumnos.

- Profesores: herramientas de autoría, herramientas de compresión y navegación, calendario de formación a docentes, y descripción y tipos de espacios virtuales de trabajo -EVT-.

Al Campus Virtual tienen acceso todos los miembros de la comunidad universitaria en los siguientes grupos: alumnos, personal docente e investigador 
y personal de administración y servicios. Una vez virtualizadas las asignaturas y generado un Espacio Virtual de Trabajo a través de un curso especial de WebCT o Moodle, el profesor es el encargado de la actualización de contenidos y, por tanto, el único administrador del curso.

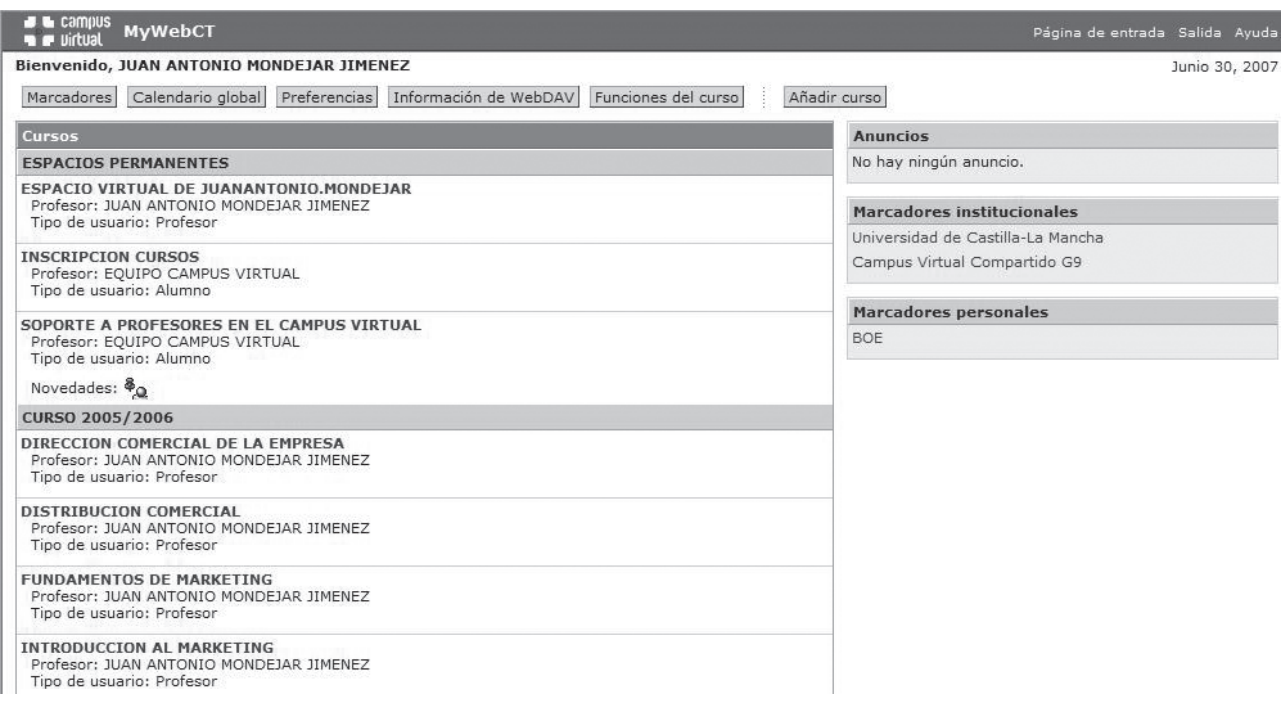

Figura 6. Ejemplo de asignaturas virtualizadas en WebCT

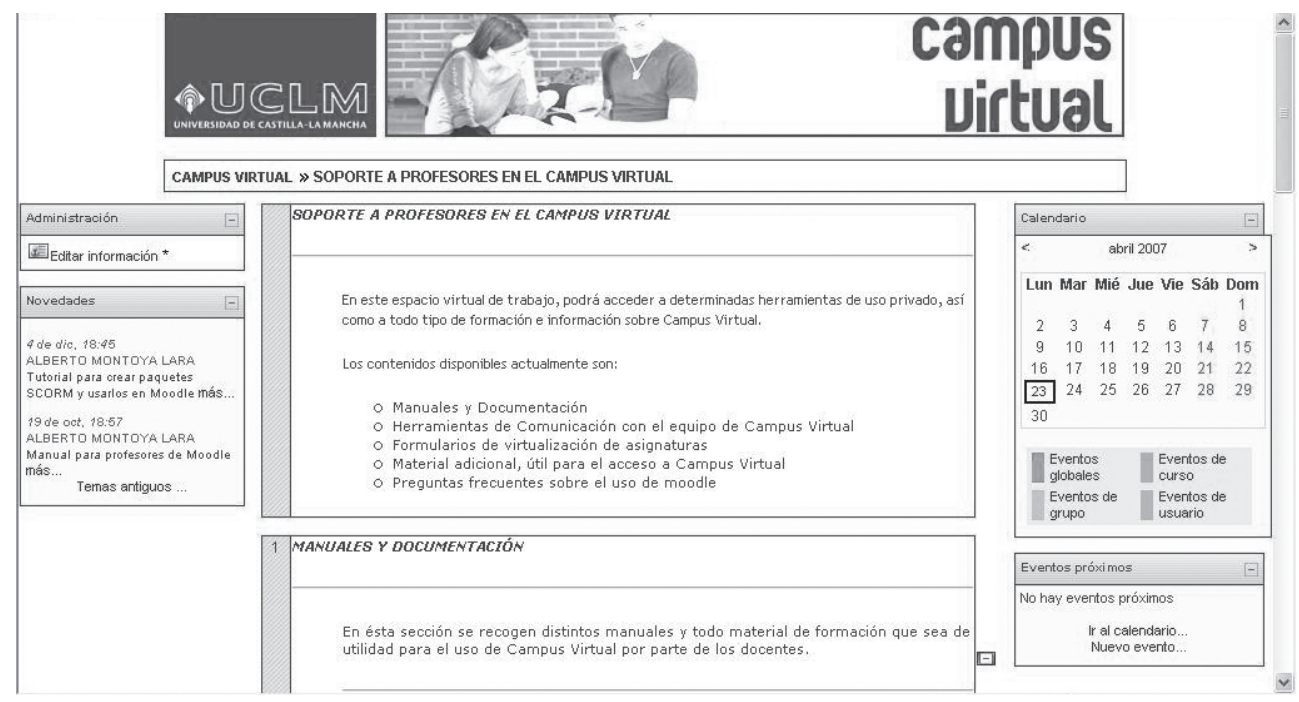

Figura 7. Menú Campus Virtual Moodle 
Si entramos más en profundidad en el espacio virtual del profesor, podemos encontrar las siguientes herramientas en los diferentes menús:

- Inicio: contiene el punto de entrada al curso.

- Guía docente: donde se ha dejado información referente al contenido de la misma, los objetivos del curso, bibliografía y datos del profesor (ubicación del despacho, teléfono de contacto, e-mail, horario de atención a alumnos, etc.). Las mencionadas guías docentes han sido elaboradas adecuando la asignatura al sistema de créditos europeos ECTS ${ }^{4}$.

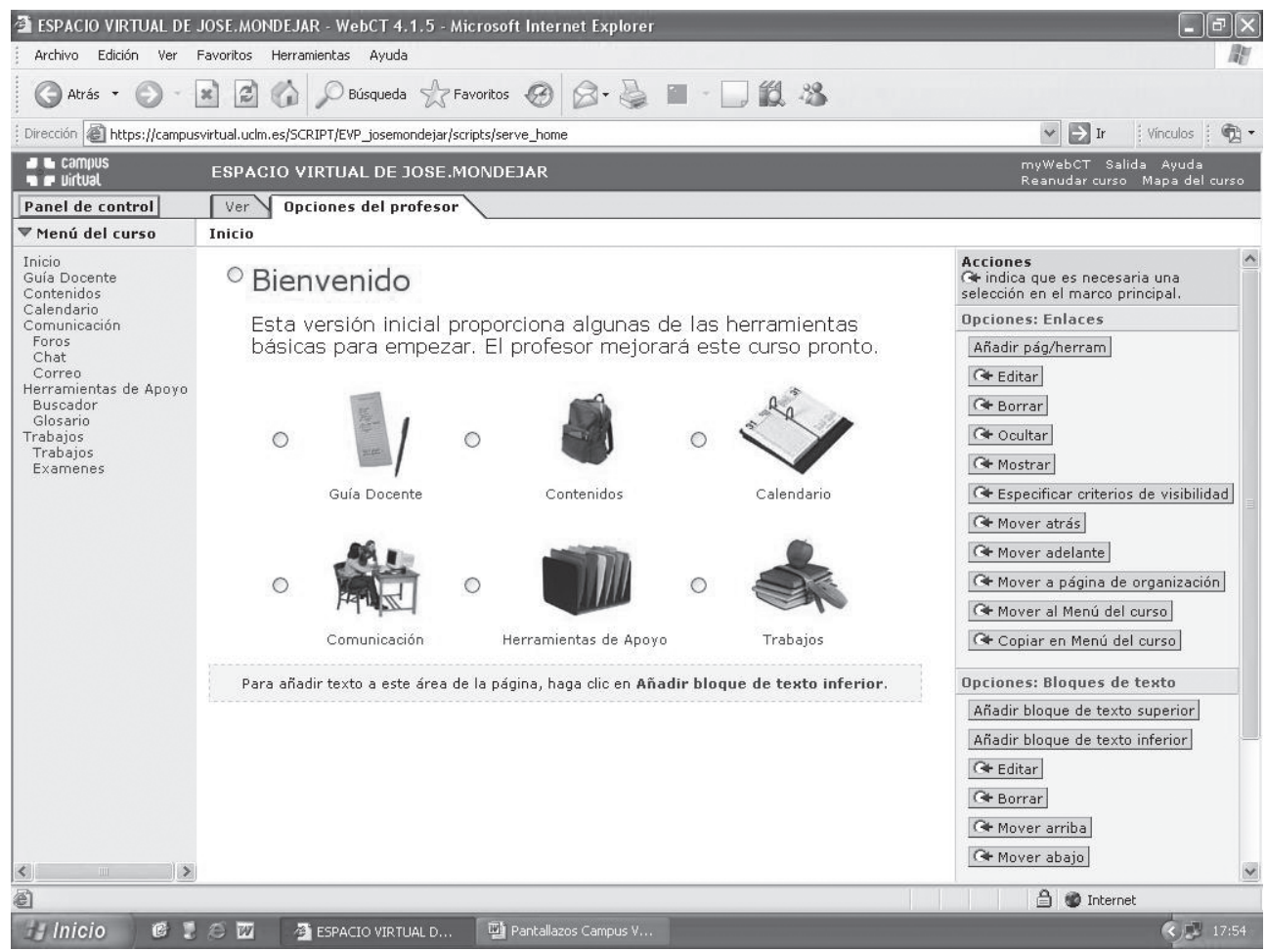

Figura 8. Menú de un curso 


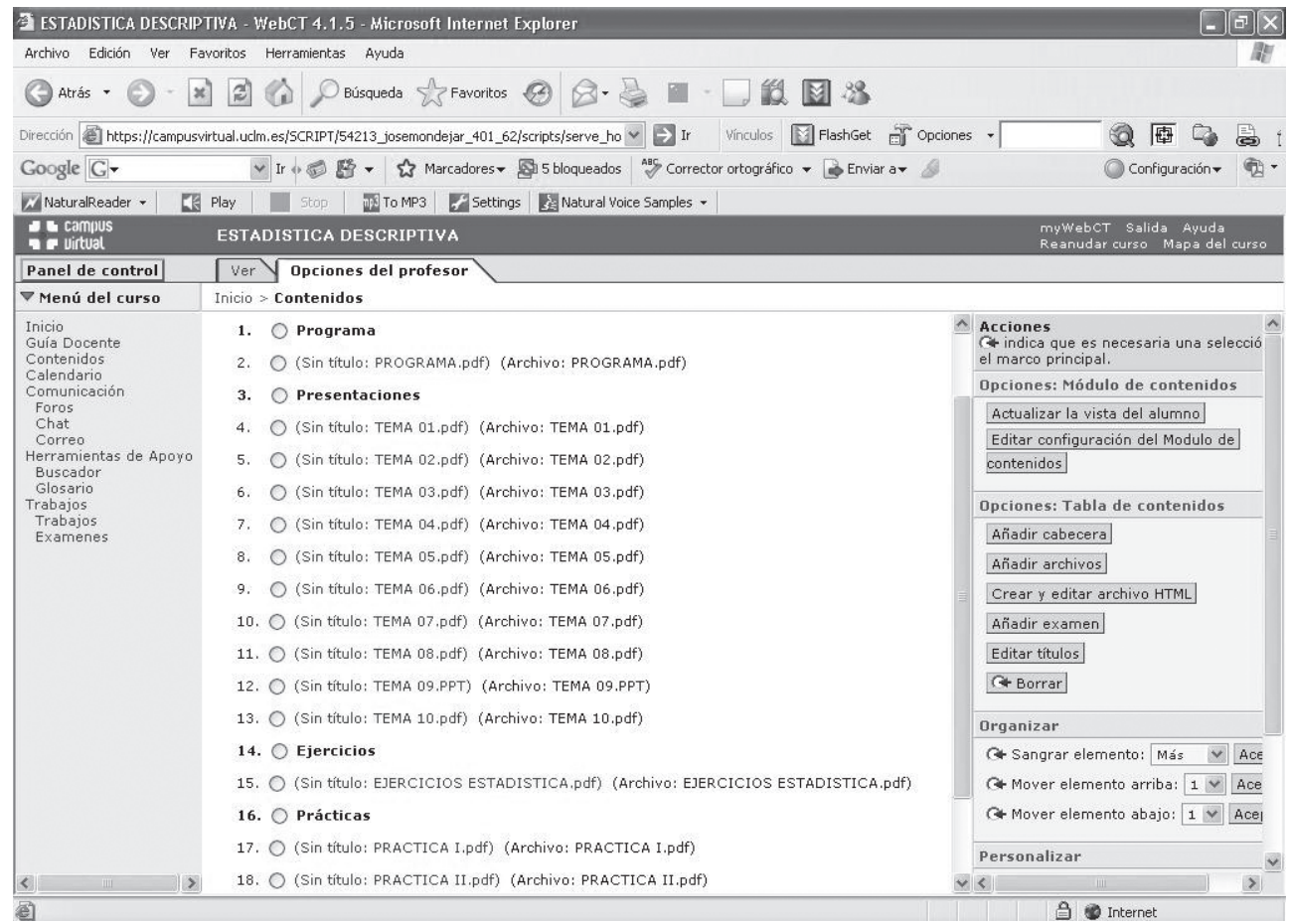

Figura 9. Contenidos asignatura estadística descriptiva

- Contenidos: En este apartado se incluyen las presentaciones de los temas de clase, así como colecciones de ejercicios y prácticas para resolver y entregar a lo largo del curso. Para la resolución de las prácticas se recomienda la utilización de las herramientas de comunicación, que veremos en los puntos siguientes. En la figura 9 podemos observar el ejemplo de contenidos para una asignatura virtualizada en el curso 2006/2007.

- Calendario: Se muestra un calendario académico donde se insertan las fechas importantes del curso, tales como clases en el aula de informática, fechas tope de entrega de prácticas, exposición de trabajos, y otras fechas de interés para los estudiantes.

- Comunicación: este apartado tiene varias herramientas de acceso: Correo, Foro y Chat, que cuenta con salas de debate especiales -por temas, por grupos e incluso salas virtuales generales, donde se pueden conectar alumnos de la misma asignatura, matriculados en diferentes Campus de la Universidad de Castilla-La Mancha, e incluso en distintas materias o especialidades-. 


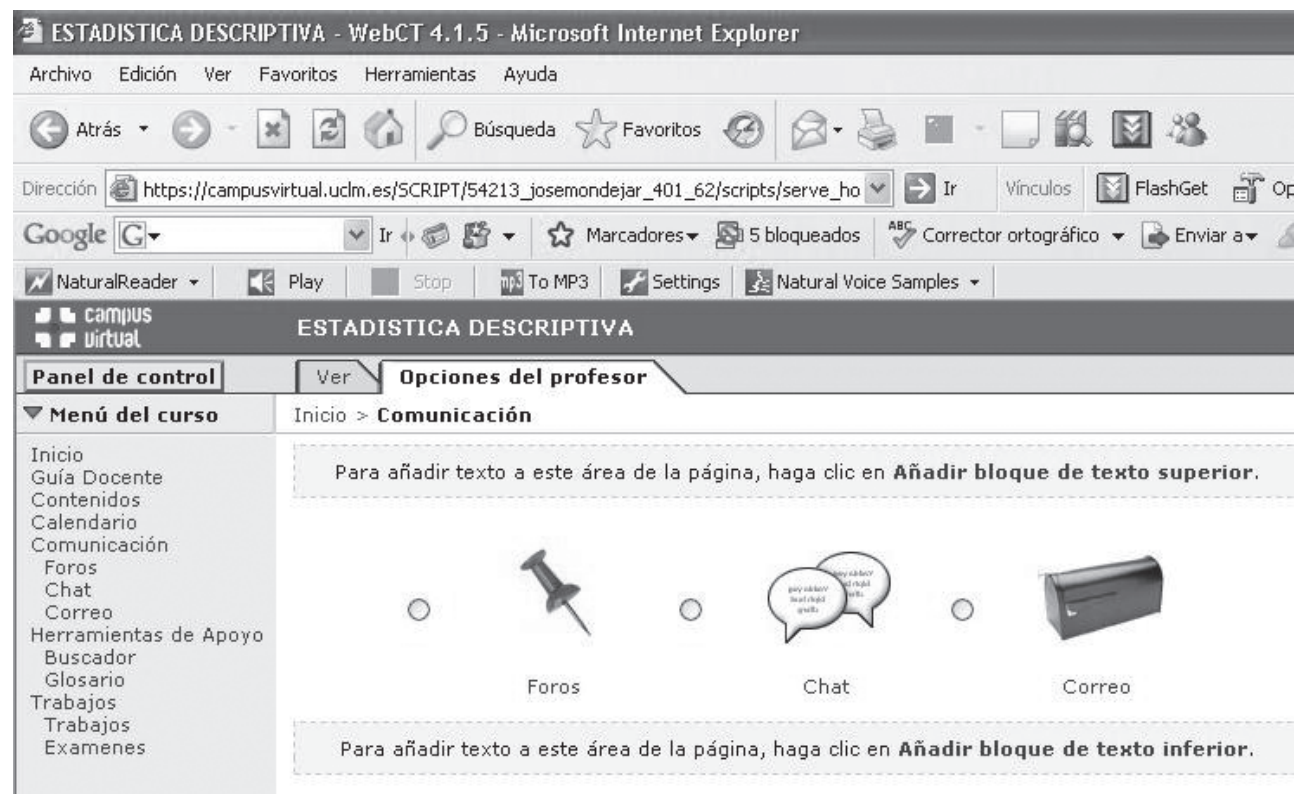

Figura 10. Herramientas de comunicación

En las distintas asignaturas se utilizan todas las herramientas de comunicación: el correo para el envío de las prácticas; el Foro para la resolución de prácticas, ya que los alumnos envían al foro sus dudas, que son respondidas por sus propios compañeros (no obstante, si pasado un tiempo prudencial no contesta algún alumno, es el profesor quien contesta a la duda); y Chat, donde los alumnos conversan de la asignatura y de otras cuestiones. En este sentido, es preciso destacar que el profesor recibe copia del Chat y foro.

- Herramientas de apoyo: Se incluye un buscador para el curso y un glosario de términos necesarios para la comprensión de la asignatura que son actualizados por el profesor durante las primeras semanas de clase.

- Trabajos: Posibilidad de entrega de trabajos que son visibles por el resto de compañeros (a veces los distintos alumnos participan en tareas de evaluación) y examen virtual, mediante esta herramienta se pueden realizar distintas pruebas de evaluación a los alumnos que pueden resolver desde las aulas de informática de la Facultad e incluso desde su propia casa. Se pueden incluir restricciones de plazo para la entrega: debido a la complejidad de los mismos y al nerviosismo que generan entre los alumnos las pruebas de evaluación, tan 
sólo se han realizado "controles tipo test" para eliminar algunos contenidos teóricos de la prueba final. Sería posible el desarrollo del examen final por parte de los alumnos, pero en muchas ocasiones los problemas de conexión obligan a realizarlos a todos los alumnos desde las aulas de informática del centro, no siendo posible su realización desde casa.

\begin{tabular}{|c|c|c|c|c|c|}
\hline $\begin{array}{l}\text { E. campus } \\
\text { ne virtuat }\end{array}$ & \multicolumn{4}{|c|}{ ESPACIO VIRTUAL DE JOSE.MONDEJAR } & \multirow[t]{2}{*}{$\begin{array}{l}\text { mywebct Salida Ayuda } \\
\text { Reanudar curso Mapa del curs. }\end{array}$} \\
\hline Panel de control & \multirow{2}{*}{\multicolumn{5}{|c|}{$\begin{array}{l}\text { Ver } \\
\text { Inicio > Panel de control básico }\end{array}$}} \\
\hline$\nabla$ Menú del curso & & & & & \\
\hline \multirow{5}{*}{$\begin{array}{l}\text { Inicio } \\
\text { Guía Docente } \\
\text { Contenidos } \\
\text { Calendario } \\
\text { Comunicación } \\
\text { Foros } \\
\text { Chat } \\
\text { Correo } \\
\text { Herramientas de Apoyo } \\
\text { Buscador } \\
\text { Glosario } \\
\text { Trabajos } \\
\text { Trabajos } \\
\text { Examenes }\end{array}$} & \multicolumn{5}{|c|}{ Panel de control básico Panel de control avanzado } \\
\hline & Añadir pág/herram & Editar pág/herram & Admin. archivos & Admin. Curso & Config. curso \\
\hline & \multirow{3}{*}{$\begin{array}{l}\text { Añada herramientas al } \\
\text { curso para evaluar a los } \\
\text { alumnos, comunicarse con } \\
\text { ellos y proporcionarles } \\
\text { contenidos del curso. } \\
\text { Desarrolle contenidos del } \\
\text { curso para los alumnos } \\
\text { con herramientas como } \\
\text { Programa y Módulo de } \\
\text { contenidos. Agrupe los } \\
\text { contenidos y las } \\
\text { herramientas del curso } \\
\text { mediante Páginas de } \\
\text { organización. }\end{array}$} & $\begin{array}{l}\text { Personalizar la Página } \\
\text { principal y cambiar el } \\
\text { contenido del curso. }\end{array}$ & \multirow[t]{3}{*}{$\begin{array}{l}\text { Añada, descargue, cambie } \\
\text { y organice los archivos del } \\
\text { curso. }\end{array}$} & \multirow{3}{*}{$\begin{array}{l}\text { Administre las fichas de } \\
\text { los alumnos y ayudantes } \\
\text { del profesor. Siga el } \\
\text { progreso de los alumnos. } \\
\text { Administre copias de } \\
\text { seguridad del curso. } \\
\text { Importe y exporte } \\
\text { contenido de IMS. }\end{array}$} & \multirow[t]{3}{*}{$\begin{array}{l}\text { Cambie el aspecto y el } \\
\text { funcionamiento del curso. }\end{array}$} \\
\hline & & $\begin{array}{l}\text { Actualice las vistas de los } \\
\text { alumnos de los Módulos de } \\
\text { contenidos del curso. }\end{array}$ & & & \\
\hline & & Actualizar la vista del alumno & & & \\
\hline
\end{tabular}

Figura 11. Panel de control básico

Por último, incluimos en las figuras 11 y 12 dos vistas del panel de control básico y avanzado del profesor donde puede realizar cambios técnicos y estéticos de la presentación del curso.

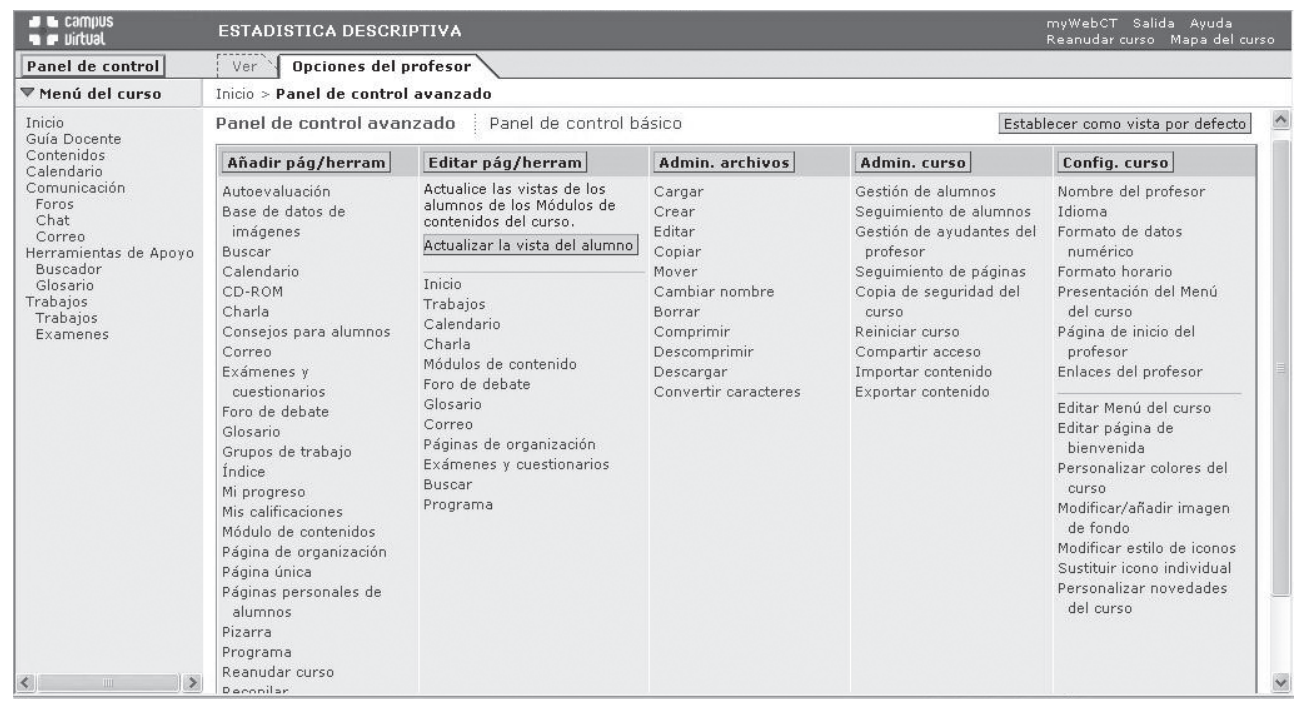

Figura 12. Panel de control avanzado 


\section{EVALUACIÓN DE LA IMPLANTACIÓN EN LA FACULTAD DE CIENCIAS SOCIALES}

Los autores han desarrollado su actividad docente, a lo largo de los últimos cursos académicos, en el marco de los diversos Proyectos de Innovación Docente de la Facultad de Ciencias Sociales de Cuenca. La experiencia acumulada en este periodo permite aportar una serie de resultados derivados de la progresiva implantación de las nuevas herramientas virtuales en el marco de la docencia reglada presencial.

Resulta obvio que la mayor parte de mejoras encaminadas a favorecer la adaptación de los estudios y el método de enseñanza-aprendizaje a las directrices del nuevo Espacio Europeo de Educación Superior suponen un importante esfuerzo tanto para los docentes como para los propios estudiantes, especialmente en los primeros años (que pueden ser considerados como "experimentales").

No obstante, con objeto de poder tener una evaluación de la implantación de estas nuevas herramientas y técnicas pedagógicas, se ha desarrollado un cuestionario que permite obtener una información verdaderamente relevante para los profesores que imparten las diversas materias, además de contribuir a la mejora en la adaptación al nuevo modelo europeo de enseñanza-aprendizaje.

El número de cuestionarios válidos en el curso académico 2006/2007 ha sido de 317, correspondientes a cinco asignaturas del área de conocimiento de Economía Aplicada (Estadística) más tres del área de Comercialización e Investigación de Mercados. En el cuestionario se ha requerido la valoración por parte de los alumnos, utilizando una escala de o a 10, de diversos aspectos relacionados con la implantación de las nuevas técnicas pedagógicas al amparo del nuevo sistema ECTS.

Los resultados más significativos, desde el punto de vista de la incorporación de las nuevas tecnologías a la enseñanza presencial, han sido los que muestra la tabla 1: 


\begin{tabular}{|l|c|}
\hline Cuestión & Puntuación Media \\
\hline Campus Virtual permite una mejor distribución de las actividades programadas & 7,63 \\
\hline Campus Virtual fomenta el trabajo en equipo & 8,74 \\
\hline El uso de Campus Virtual es fomentado por el profesor & 9,17 \\
\hline Mejora mi capacidad de planificación y organización en el desarrollo de la materia & 6,58 \\
\hline Considero adecuada la distribución entre actividades presenciales y virtuales & 8,62 \\
\hline Uso habitualmente las tutorías virtuales & 5,73 \\
\hline Valoración de las herramientas de comunicación virtuales (foto, Chat, etc...) & 8,38 \\
\hline Son de utilidad los materiales de la asignatura disponibles en Campus Virtual & 9,60 \\
\hline Valoración de los métodos docentes empleados & 8,12 \\
\hline
\end{tabular}

Tabla 1. Principales resultados de la experiencia UCLM

\section{CONCLUSIONES}

Los entornos virtuales de aprendizaje (WebCT y Moodle) están orientados a gestión de contenidos educativos, y tienen como finalidad ser una herramienta de apoyo que complete las explicaciones del docente en el aula. Estas características compartidas con la plataforma Red-C@mpus son ampliadas con la posibilidad de interrelacionarse con los alumnos en la red: gestionar cursos, programar tareas o resolver dudas en tutorías virtuales mediante Chats o foros. Las posibilidades de estas plataformas on-line son muy variadas y su utilización no entraña grandes dificultades.

Analizando las modificaciones que el Espacio Europeo de Educación Superior supone para la docencia universitaria, observamos el cambio de protagonismo en el proceso de enseñanza-aprendizaje. Según el proceso de Bolonia, el profesor adopta el papel de orientador del alumno, y transforma a éste último en el verdadero protagonista del aprendizaje. Es por ello que las nuevas tecnologías de la información desempeñan un rol básico en este proceso. Sin su utilización no podría realizarse la renovación de las metodologías pedagógicas.

La enseñanza virtual, o e-learning, es una de las ventajas que proporcionan las nuevas tecnologías, sin embargo, en este análisis que aquí realizamos, no nos centramos en sus usos y beneficios sino que pretendemos fijarnos en las plataformas on-line que facilitan el aprendizaje; en las herramientas orientadas a la colaboración entre profesor y estudiante y en lo fácil que resulta, para ambos, utilizarlas. Los 
entornos Moodle y WebCT (aunque existen muchas otras plataformas), disponen de funciones y herramientas potentes y seguras, que mejoran ampliamente los antiguos programas, además son herramientas destinadas únicamente al aprendizaje del alumno, puesto que no contemplan las posibilidades de matriculación ni demás tramites administrativos, que sí se pueden realizar en Red-C@mpus.

Entre los puntos fuertes de los entornos virtuales de aprendizaje respecto a las anteriores plataformas destacan:

- Independencia geográfica y horaria: el aprendizaje se puede realizar desde cualquier ordenador con conexión a Internet y a cualquier hora.

- Existencias de diversas plataformas para diseñar los cursos.

- Un mayor incremento de la comunicación alumno-profesor, a más de la ya existente.

- $\quad$ Mayor disponibilidad por parte de alumnos y profesores.

- $\quad$ Facilidad de uso.

- Flexibilidad didáctica y técnica: estas plataformas permiten adecuar los cursos a las necesidades de alumnos y docentes.

- Permite el seguimiento, control y registro del alumnado.

Si tenemos en cuenta los principales puntos débiles, que empeoran la situación existente destacamos:

- Problemas de acceso y disponibilidad de recursos, de especial relevancia cuando nuestros alumnos regresan a sus municipios de origen los fines de semana: éstos no disponen de líneas de conexión a alta velocidad (ADSL), lo que complica la gestión de grandes volúmenes de información.

- $\quad$ Necesidad de equipos potentes para trabajar adecuadamente. Las anteriores plataformas eran utilizadas mayoritariamente para consultar el expediente.

- Se requiere un esfuerzo adicional por parte de alumnos y profesores en el diseño de materiales.

- Inexistencia de norma de calidad de formación, por tanto, el incremento en materiales no siempre supone incremento en la calidad docente.

- $\quad$ La experiencia de la plataforma Red-C@mpus en la Universidad de CastillaLa Mancha, tras ocho cursos académicos de implantación, ha tenido un 
uso especialmente dirigido al apartado administrativo, de calificación de estudiantes, etc. pero, sin embargo, no se ha desarrollado como una auténtica herramienta de e-learning para la enseñanza universitaria.

Tal y como se aprecia en los resultados derivados de la encuesta a estudiantes durante el último curso académico de implantación de estas técnicas, se ha obtenido una alta valoración por parte de los estudiantes, si bien la participación de 317 alumnos no se corresponde con el total de matriculados sino sólo aquellos que asisten presencialmente a clase. Es preciso destacar aquí el interés por conseguir la información relativa a aquellos estudiantes que siguen la asignatura exclusivamente de forma virtual, al no asistir a clase por motivos laborales o de otra índole. Se trata, entonces, de una línea futura de investigación a tomar en consideración.

No obstante, la presencia de las nuevas tecnologías ofrece una alternativa a la docencia orientada a la interactividad, la colaboración y la motivación. Las plataformas virtuales de aprendizaje son herramientas muy útiles que facilitan las tareas al profesor y al estudiante. La evolución de estas aplicaciones, que gestionan contenidos educativos, ha ido pareja al desarrollo de la tecnología lo que ha mejorado sus utilidades y ventajas. Los entornos virtuales constituyen una alternativa en el ámbito del aprendizaje virtual y que fomenta el aprendizaje colaborativo. Estas utilidades complementan la enseñanza presencial con la virtual fomentando la calidad de la enseñanza.

\section{Nota}

Los autores desean agradecer los comentarios de los evaluadores anónimos, que han contribuido a mejorar la versión final del artículo. Obviamente, la responsabilidad de los contenidos es exclusiva de los autores.

\section{NOTAS}

1 La asociación dispone de una completa página web en Internet: www. uni-g9.net.

2 Para una exposición más detallada puede consultarse Mondéjar, Gómez y Lorenzo (2006).

3 El uso de la plataforma libre Moodle sólo es posible desde su aplicación en el segundo semestre del curso 2006/2007. 
4 La elaboración de estas guías docentes está enmarcada dentro de los Proyectos de Innovación Docente de las asignaturas de $1^{\mathrm{o}}$ y $2^{\mathrm{o}}$ curso de la licenciatura en Administración y Dirección de Empresas y la diplomatura en Relaciones Laborales, antes mencionados.

\section{REFERENCIAS BIBLIOGRÁFICAS}

Acosta Lugo, W. (2004). Aproximación a elearning y el Aprendizaje a Distancia. Learning Review, 1,junio-julio. [en línea] Disponible en: www.learningreview.com. ar. [consulta 2007, 26 de marzo].

Barchino Plata, R. (2005). Panorámica institucional del proceso de evaluación en la enseñanza y aprendizaje electrónico. RIED - Revista Iberoamericana de Educación a Distancia, 8 (1), 83-104.

Belanger, F.; Jordan, D. H. (2000). Evaluation and implementation of distance learning: technologies, tools and techniques. Londres: Idea Group Publishing.

Buzón García, O. (2005). La incorporación de plataformas virtuales a la enseñanza: una experiencia de formación online basada en competencias. Revista Latinoamericana de Tecnología Educativa, 4 (1), 77-98. [en línea] Disponible en: http://www.unex.es/ didactica/RELATEC/sumario 4 1.htm. [consulta 2007, 26 de marzo].

Carabantes, D.; Carrasco, A.; Alves, J. D. (2005).Lainnovaciónatravés deentornos virtuales de enseñanza y aprendizaje. RIED - Revista Iberoamericana de Educación a Distancia, 8 (1), 105-126.

Casas Armengol, M.; Stojanovic, L. (2005). Innovación y virtualización progresivas de las universidades iberoamericanas hacia la sociedad del conocimiento. RIED - Revista Iberoamericana de Educación a Distancia, 8 (1), 127-146.

Cebrián, M. (2003). Enseñanza virtual para la innovación universitaria. Madrid: Narcea.
Duart, J. M.; Salomón, L.; Llara, P. (2006). La Universitat Oberta de Catalunya (UOC): innovación educativa y tecnológica en educación superior. RIED - Revista Iberoamericana de Educación a Distancia, 9 (1 y 2), 315-344.

González Díaz-Caneja, I.; López García, A. M.; Chasco Irigoyen, C. (2003). El elearning en la universidad española. XVII Reunión Anual Asepelt-España. Universidad de Almería.

Kearsley, G. (2000). On line education. Learning and teaching in cyberspace. Toronto: Wadsworth.

Lara Navarra, P. (coord.) (2005). Uso de contenidos digitales: tecnologías de la información, sociedad del conocimiento y universidad. Revista de Universidad y Sociedad del Conocimiento (RUSC), 2 (2), número monográfico. UOC.

Mayor, J.; Suengas, A.; González-Marqués, J. (1993). Estrategias metacognitivas. Aprender a aprender $y$ aprender $a$ pensar. Madrid: Ed. Síntesis.

Mondéjar Jiménez, J.; Mondéjar Jiménez, J. A.; Vargas Vargas, M. (2006). Implantación de la metodología elearning en la docencia universitaria: una experiencia a través del proyecto Campus Virtual. Revista Latinoamericana de Tecnología Educativa, 5 (1), 59-71. [en línea] Disponible en: http://www.unex. es/didactica/RELATEC/sumario 5 1.htm [consulta 2007, 26 de marzo].

Mondéjar Jiménez, J. A.; Gómez Borja, M. A.; Lorenzo Romero, C. (2006). RedC@mpus: una herramienta de apoyo en la implantación de metodologías ECTS 
en la Universidad de Castilla-La Mancha. I Jornadas Nacionales de Intercambio de Experiencias Piloto de Implantación de Metodologías ECTS, Universidad de Extremadura, Badajoz.

Pls Ramboll Management (2004). Studies in the Context of the E-learning Initiative: Virtual Models of European Universities (LOT 1). Draft Final Report to the EU Commission, DG Education \& Culture Virtual Models of Universities. [en línea] Disponible en: www.elearningeuropa. info/extras/pdf/virtual models.pdf [consulta 2007, 26 de marzo].
Rangecroft, M.; Gilroy, P.; Long, P. (1999). What is important to distance education students. Open Learning, 17-24.

Valverde Berrocoso, J. (2002). Herramientas decomunicación sincrónicayasincrónica, en: Cabero, J. y Aguaded, J. I. Educar en red: Internet como recurso para la educación, 57-81. Málaga: Aljibe.

Wilson, R. L.; Weiser, M. (2001). Adoption of asynchronous learning tools by traditional full-time students: a pilot study. Information Technology and Management, 2, 363-375.

\section{PERFIL ACADÉMICO Y PROFESIONAL DE LOS AUTORES}

Juan Antonio Mondéjar Jiménez: Doctor y licenciado en Administración y Dirección de Empresas por la Universidad de Castilla-La Mancha. Diplomado en Estudios Avanzados en Comercialización e Investigación de Mercados por la misma Universidad. Profesor del Área de Comercialización e Investigación de Mercados en el Departamento de Administración de Empresas de la Facultad de Ciencias Sociales de Cuenca (Universidad de Castilla-La Mancha) y profesor consultor de la Universidad Oberta de Cataluña (UOC).

E-mail: JuanAntonio.Mondejar@uclm.es

José Mondéjar Jiménez: Doctor Europeo en Economía y licenciado en Administración y Dirección de Empresas por la Universidad de Castilla-La Mancha. Diplomado en Estudios Avanzados en Estadística por la misma Universidad. Profesor del Área de Economía Aplicada (Estadística) en la Facultad de Ciencias Sociales de Cuenca (Universidad de Castilla-La Mancha). Líneas principales de investigación: Indicadores sintéticos, espacio de estados, desarrollo rural y docencia en entornos virtuales.

E-mail: Jose.Mondejar@uclm.es

Manuel Vargas Vargas: Doctor en Economía por la Universidad de CastillaLa Mancha y licenciado en Matemáticas por la Universidad de Granada. Profesor Titular de Universidad del Área de Economía Aplicada (Estadística) en la Facultad de 
Ciencias Sociales de Cuenca (Universidad de Castilla-La Mancha). Líneas principales de investigación. Estadística bayesiana, espacio de estados, series temporales y docencia en entornos virtuales.

E-mail: Manuel.Vargas@uclm.es

DIRECCIÓN DE LOS AUTORES

Universidad de Castilla-La mancha

Facultad de Ciencias Sociales

Área de Estadística

Avda. de los Alfares, 44

16.071-CUENCA (España)

Fecha de recepción del artículo: 11/06/07

Fecha de aceptación del artículo: 24/09/07 